\title{
The use of monocyte subset repartitioning by flow cytometry for diagnosis of chronic myelomonocytic leukaemia
}

\author{
Aarya Murali $\mathbb{D}^{1,2}$, Donna Cross $^{2}$ and Peter Mollee ${ }^{1,2}$
}

Dear Editor,

We note with interest Pophali et al.'s ${ }^{1}$ paper examining the value of monocyte subset repartitioning by multiparametric flow cytometry (MFC) to distinguish chronic myelomonocytic leukaemia (CMML) from other causes of monocytosis. The original paper by Selimoglu-Buet et al. ${ }^{2}$ in 2015 noted a relative predominance of classical or MO1 monocytes (CD14+/CD16-) at the expense of MO2 (CD14low/CD16+) and MO3 (CD14-/CD16+) monocytes in patients with CMML. These authors suggested that an MO1 percentage cut-off of $>94 \%$ could predict the diagnosis of CMML with high sensitivity and specificity (both $>90 \%$ ) whereas Pophali et al. were unable to replicate these findings, calling into question the utility of flow cytometry to distinguish the aetiology of monocytosis in a real-world setting. We wish to add data from our own experience to shed further light on this issue.

In this study, we assessed peripheral blood samples from 35 patients presenting with a monocytosis (absolute monocyte count $>1 \times 10^{9} / \mathrm{L}$ ) in a tertiary referral hospital in Brisbane, Australia. The patients' final clinical diagnosis was extracted from the medical record by two clinicians (A.M. and P.M.) blinded to the results of the flow cytometry. Ethical approval for this study was obtained from the Metro South Hospital and Health Service Human Research Ethics Committee [HREC/2020/QMS/65502]. A detailed description of the methods of flow cytometry applied in this study is available in supplementary materials.

Of the 35 patients included, 13 patients had CMML and 4 patients were diagnosed with another underlying myeloid neoplasm: one patient with myelodysplastic syndrome

Correspondence: Aarya Murali (aarya.murali@health.qld.gov.au)

${ }^{1}$ School of Medicine, University of Queensland, Brisbane, QLD, Australia

2Department of Haematology, Princess Alexandra Hospital, Brisbane, QLD,

Australia
(MDS), one patient with myeloproliferative neoplasm-not otherwise specified (MPN-NOS), one patient with multiple myeloma and one patient with acute myeloid leukaemia with myelodysplasia related changes (AML-MRC). Table 1 summaries the individual characteristics of the 13 patients diagnosed with CMML (note: next-generation sequencing panels were not performed during the time frame of this study).

Furthermore, six patients had a reactive monocytosis in the setting of autoimmune disease, including granulomatosis with polyangiitis (GPA) (two patients), rheumatoid arthritis (RA) (one patient), IgG4 disease (one patient), mixed connective tissue disease (MCTD) (one patient), and polyarticular gout (one patient). Lastly, 12 cases of reactive monocytosis were attributed to infective and inflammatory causes.

Among our 13 patients with CMML, 7 cases $(53.8 \%)$ had an MO1 percentage $>94 \%$. Among the six patients with CMML who had an MO1 percentage $\leq 94 \%$, five patients had another underlying inflammatory or autoimmune condition, including asbestosis (patient ID 10); anti-phospholipid antibody syndrome (patient ID 15); granuloma annulare (patient ID 16); urosepsis, ocular shingles and polycythaemia rubra vera (patient ID 20); and lastly, polyarteritis nodosa (patient ID 21). These patients had an MO2 fraction which ranged from 4 to $35 \%$ (Table 1). All but two patients with CMML were treatment naïve at the time that monocyte subset repartitioning was performed. Only one patient met criterion for therapy-related MDS/MPN.

In comparison, only $4(18.2 \%)$ among the 22 cases of non-CMML were identified to have an MO1 percentage $>94 \%$-this included two patients with autoimmune disease (GPA, MCTD), one patient with MDS and one patient with recurrent infections. 
Table 1 Characteristics of patients with CMML $(n=13)$.

\begin{tabular}{|c|c|c|c|c|c|c|c|c|}
\hline Patient ID & CMML subtype & $\begin{array}{l}\text { Bone marrow } \\
\text { cytogenetics }\end{array}$ & AMC & M01\% & MO2\% & MO3\% & $\begin{array}{l}\text { Previous treatment } \\
\text { for CMML }\end{array}$ & $\begin{array}{l}\text { Meeting criterion for } \\
\text { t-MDS/t-MPN }\end{array}$ \\
\hline 10 & CMML-1 & $46 X Y, N A D$ & 1.24 & 93 & 6 & $<1$ & No & No \\
\hline 11 & CMML-1 & $46 X X, N A D$ & 8.15 & 96 & 4 & $<1$ & No & No \\
\hline 15 & CMML-2 & $46 X X, N A D$ & 1.4 & 74 & 21 & 4 & No & No \\
\hline 16 & CMML-2 & $46 X Y, N A D$ & 0.69 & 35 & 4 & 40 & $Y_{e s}{ }^{a}$ & No \\
\hline 17 & $C M M L^{b}$ & $\mathrm{~N} / \mathrm{A}$ & 2.38 & 95 & 4 & 1 & No & No \\
\hline 18 & $\mathrm{CMML}^{\mathrm{b}}$ & N/A & 2.19 & 95 & 4 & 1 & No & No \\
\hline 20 & CMML-2 & Not done & 5.05 & 94 & 6 & $<1$ & No & Yes $^{c}$ \\
\hline 21 & CMML-1 & Not done & 5.64 & 58 & 35 & 7 & No & No \\
\hline 23 & CMML-2 & $46 X X, N A D$ & 36.51 & 98 & $<1$ & $<1$ & Yes $^{d}$ & No \\
\hline 25 & $C M M L-1^{b}$ & $\mathrm{~N} / \mathrm{A}$ & 3.66 & 95 & 2 & 2 & No & No \\
\hline 28 & $\begin{array}{l}\text { CMML (subtype not } \\
\text { specified) }\end{array}$ & $46 X Y, N A D$ & 4.44 & 97 & 1 & 2 & No & No \\
\hline 31 & $\mathrm{CMML}^{\mathrm{e}}$ & N/A & 7.48 & 74 & 13 & 12 & No & No \\
\hline 32 & $C M M L^{f}$ & N/A & 6.48 & 95 & 2 & 2 & No & No \\
\hline
\end{tabular}

${ }^{a}$ Monocyte flow cytometry was performed at Cycle 1 Day 7 of Azacitadine for treatment for CMML.

${ }^{b}$ Diagnosis of CMML was made at external institution; unable to locate report of original bone marrow aspirate and trephine.

cPrevious breast cancer (1994) treated with lumpectomy and radiation therapy; previous diagnosis of polycythaemia rubra vera, treated with hydroxyurea.

${ }^{\mathrm{d}} \mathrm{CMML}$ previously treated with hydroxyurea-ceased after splenectomy for splenic infarction, approximately 8 months prior to monocyte flow cytometry.

eBone marrow aspirate and trephine were not performed due to significant co-morbidities including dementia.

fone marrow aspirate and trephine were not performed as declined by patient.

There was no significant correlation between an MO1 percentage cut-off of $>94 \%$ and a diagnosis of CMML $(p=0.057)$. We also examined the utility of the MO3 percentage cut-off of $<1.13 \%$, established by Hudson et al. ${ }^{3}$ in their paper from 2018. Among our 13 cases of CMML, six patients $(46.2 \%)$ were noted to have an MO3 percentage $<1.13 \%$. Meanwhile, only 8 out of 22 nonCMML patients $(36.4 \%)$ had MO3 percentage $<1.13 \%$. There was no association between MO3 percentage and the diagnosis of CMML $(p=0.724)$. Hence, our study confirms the limitations of peripheral blood MFC in the diagnosis of CMML as highlighted by Pophali et al. Results from other publications assessing this issue are summarised in Supplementary Table 1 (refs. ${ }^{1-4}$ ).

Using the MO1 and MO3 percentage cut-offs previously established, we were unable to reliably diagnose CMML. In fact, in our study there was a distinct subset of four CMML patients with MO1 percentage values significantly lower than the cut-off of $94 \%$. Notably, among this were two patients with CMML-2 (with MO1 percentages of 35 and $74 \%$ ) and one patient with CMML which transformed to secondary AML (with MO1 percentage of 58\%). Similar subsets of CMML patients with low MO1 percentages were also reported by Hudson et al. and Selimoglu-Buet et al. Furthermore, Picot et al. also alluded to the changes observed in $\mathrm{MO} 1, \mathrm{MO} 2$ and $\mathrm{MO} 3$ distribution depending on when the testing was performed ( 24 vs $48 \mathrm{~h}$ after harvesting). Picot et al. commented that when the analysis was performed at $48 \mathrm{~h}$ after collection, even in patients with CMML, the monocyte subset profile resembled that seen in reactive monocytosis. However, among our six patients with CMML noted to have an $\mathrm{MO} 1$ percentage $\leq 94 \%$, the majority of samples, i.e. five, were analysed within $24 \mathrm{~h}$ and one sample was processed between 24 and $48 \mathrm{~h}$ from collection. Therefore, in our real-world data, time to analysis does not appear to have been a significant factor that influenced results.

In summary, there is a clinical need to develop accurate, reliable, and cost-effective methods to distinguish monocytosis due to CMML from other reactive entities. Although monocyte subset repartitioning has shown promise in previous works, our study highlights some of its limitations in a real-world setting. In agreement with Pophali et al., we suggest that more research with larger sample sizes is required before monocyte subset analysis can be confidently applied to discriminate reactive monocytosis from CMML.

\section{Conflict of interest}

For each of the authors, we have the following conflicts of interest and financial relationships to disclose: A.M. (first author, corresponding author): none. D.C. (second author): none. P.M. (third author): Janssen (membership on Board of Directors or advisory committees and Research Funding), BMS/ Celgene (membership on Board of Directors or advisory committees), Amgen (membership on Board of Directors or advisory committees), Takeda (membership on Board of Directors or advisory committees), Pfizer (membership on Board of Directors or advisory committees) and Caelum (membership on Board of Directors or advisory committees).

\section{Publisher's note}

Springer Nature remains neutral with regard to jurisdictional claims in published maps and institutional affiliations.

Supplementary Information accompanies this paper at (https://doi.org/ 10.1038/s41408-020-00401-3). 
Received: 27 August 2020 Revised: 30 November 2020 Accepted: 8 December 2020

Published online: 07 January 2021

\section{References}

1. Pophali, P. A. et al. Practical limitations of monocyte subset repartitioning by multiparametric flow cytometry in chronic myelomonocytic leukemia. Blood Cancer J. 9, 65 (2019).
2. Selimoglu-Buet, D. et al. Characteristic repartition of monocyte subsets as a diagnostic signature of chronic myelomonocytic leukemia. Blood 125, 3618-3626 (2015).

3. Hudson, C. A., Burack, W. R., Leary, P. C. \& Bennett, J. M. Clinical utility of classical and nonclassical monocyte percentage in the diagnosis of chronic myelomonocytic leukemia. Am. J. Clin. Pathol. 150, 293-302 (2018).

4. Picot, T. et al. Evaluation by flow cytometry of mature monocyte subpopulations for the diagnosis and follow-up of chronic myelomonocytic leukemia. Front. Oncol. 8, 109 (2018). 